\title{
Antiproliferative activities of some 7-hydroxy-3- aryloxy-2-trifluoromethyl-4H-4-chromenone derivatives against 60 human cancer cell lines
}

\author{
A. O. Prykhod'ko, G. G. Dubinina, V. P. Khilya ${ }^{1}$, S. M. Yarmoluk \\ Institute of Molecular Biology and Genetics, National Academy of Sciences of Ukraine \\ 150 Acad. Zabolotnoho str., Kyiv, 03143, Ukraine \\ Kyiv National Taras Shevchenko University \\ 64 Volodymyrska str., Kyiv, 01033, Ukraine
}

\begin{abstract}
234 derivatives of 7-hydroxy-3-aryloxy-2-trifluoromethyl-4H-4-chromenones were synthesized and tested for antitumor activity in vitro against human cancer cell lines in NCI (National Cancer Institute, USA) bioassay. It was shown high cytostatic and cytotoxic activity for the tested compounds $1-8$ (GIso 3.44-41.1 $\mu \mathrm{M}$ and $L \mathrm{C}_{50}$ from $49.6 \mu \mathrm{M}$ ). The relationship between structures of the tested compounds and their antiproliferative activities is discussed.
\end{abstract}

Introduction. Isoflavonoids are a large group of naturally occuring phenolic compounds ubiquitously distributed in the plant kingdom. The biological activity of isoflavonoids is related to their antioxidative effects $\{1-3\}$ and influences tumor cell proliferation, differentiation and apoptosis [4-8]. Antiproliferative activities of isoflavonoids include inhibition of protein tyrosine kinase [9-12], DNA topoisomerase I and II $[13,14]$, protein kinase $\mathrm{C}$ and phosphoinositol kinase [15-17], casein kinase II [18]. Inhibition of cyclindependent kinases has been also described [19]. The synthetic flavonoid flavopiridol (phase I clinical and pharmacokinetic trial) has been shown to be a potent inhibitor of cdc2 kinase activity (fig. 1) [20, 21 ].

During last years we were interested in the synthesis of isoflavonoids aimed at finding compounds with biological activity $[22,23]$. The present paper deals with the synthesis of a combinatorial series of 7-hydroxy-3-aryloxy-2-trifluoromethyl-4H-4-chromenone derivatives as potential antitumor agents.

Materials and Methods. Chemical synthesis of the library of 234 chromenone derivatives was per-

(C) A. O. PRYKHOD'KO, G. G. DUBININA, Y. P. KHILYA, S. M. YARMOLUK, 2004 formed by modification of the methods reported before [22-24]. Structure and purity of synthesized compounds were confirmed by ' $\mathrm{H}$ NMR spectroscopy. The spectra werc obtained with Varian VXR-300 NMR spectrometer at $300 \mathrm{MHz}$.

The synthesized compounds were tested for cytotoxic and antitumor activity in vitro in collaboration with National Cancer Institute of the U.S.A. (http://dtp.nci.nih.gov/index.html). The calculated measurement of effect was Percentage Growth (PG). The effect of the compound on a cell line was calculated according to the following two expressions:

$$
\begin{aligned}
& \text { If }\left(\text { Mean } O D_{\text {test }}-\text { Mean } O D_{\text {tzero }}\right) \geq 0 \text {, then } \\
& P G=100 \cdot\left(\text { Mean } O D_{\text {test }}-\text { Mean } O D_{\text {tzero }}\right) / \text { Mean } \\
& \left.O D_{\text {crrl }}-\text { Mean } O D_{\text {tzero }}\right) ; \\
& \text { If }\left(\text { Mean } O D_{\text {test }}-\text { Mean } O D_{\text {tzero }}\right)<0 \text {, then } \\
& P G=100 \cdot\left(\text { Mean } O D_{\text {lest }}-\text { Mean } O D_{\text {tzero }}\right) / \text { Mean } \\
& O D_{\text {tzero }}
\end{aligned}
$$

where Mean $O D_{\text {tzero }}$, Mean $O D_{\text {test }}$ and Mean $O D_{\text {cirl }}$ are the averages of optical density measurements of SRB-derived color just before cells exposure to the tested compounds, after 48 hours of exposure, and after 48 hours without any exposure of cells to the 


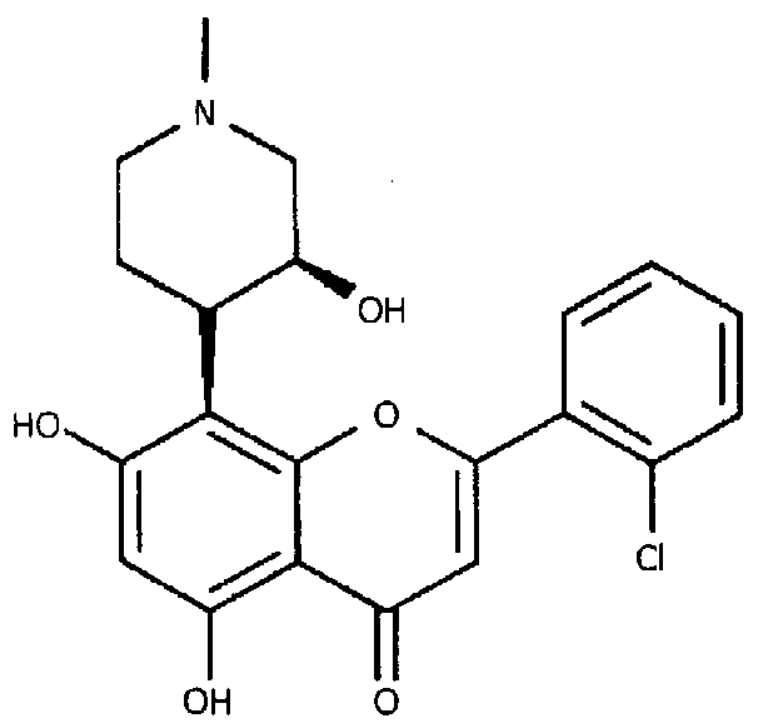

Fig. 1. Structure of flavopiridol (FLAP or NSC 649890) stage the pre-screening of all 234 compounds was carried out against 3 human cancer cell lines, MCF7 (Breast cancer), NCI-H460 (Non-small cancer cell lung) and SF-268 (CNS), at $10^{-4} \mathrm{M}$ concentration. Compounds $1-8$ (fig. 2) demonstrated antiproliferative activity ( $\mathrm{PG} \leq 32 \%$ ) and were selected for the advanced testing against 60 human cancer cell lines at five different concentrations $\left(10^{-8}-10^{-4} \mathrm{M}\right)$. Experimental data for compounds $1-8$ in representative cell lines are shown in tables 1, 2, Tested compounds $1-8$ were found to inhibit proliferation of tumor cells in the range of $\mathrm{GI}_{50} 3.44-41.1 \mu \mathrm{M}$, and exhibited cytotoxic activity against cancer cell lines ( $\mathrm{LC}_{50}$ from $49.6 \mu \mathrm{M}$ ). A number of cell lines including RPMI-8226 Leukemia, SK-MEL-5 Melanoma, T-47D Breast cancer and HCT-116 Colon cancer showed significant sensitivity to these compounds.

234 tested compounds containes various substituents at $\mathrm{C}-3, \mathrm{C}-7$ and $\mathrm{C}-8$ positions. Active<smiles>COc1cc(C(F)(F)F)c(-c2ccccc2Cl)c(=O)c2ccc(O)cc12</smiles><smiles>Oc1ccc2c(c1CN1CCCCCC1)OC(C(F)(F)F)C(Oc1ccc3ccccc3c1)[C@H]2O</smiles><smiles></smiles>
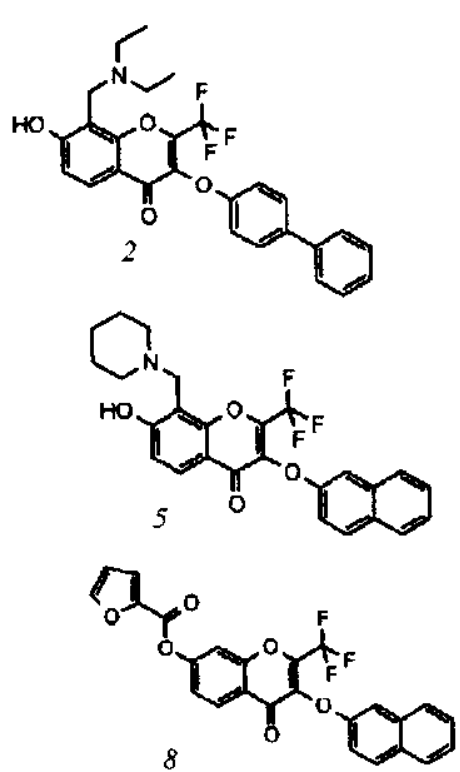
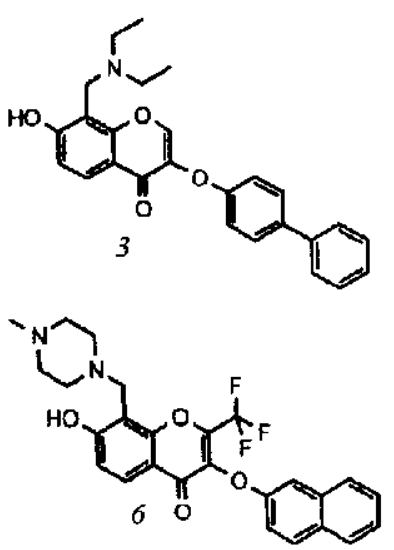

Fig. 2. Structures of cytotoxic antitumor 7-hydroxy-3-aryloxy-2-trifluoromethyl-4H-4chromenone derivatives $1-8$ selected after pre-screening tested compounds, respectively. The response parameters $\mathrm{GI}_{50}$, TGI, $\mathrm{LC}_{50}$ are interpolated values representing the concentrations at which $P G$ is $+50,0$ and -50 , respectively.

Results and Discussion. The combinatorial library of 234 derivatives of 7-hydroxy-3-aryloxy-2trifluoromethyl-4H-4-chromenone was synthesized and tested for antitumor activity in vitro against human cancer cell lines in NCI bioassay. At the first compounds 2-8 containes bulky hydrophobic substituent ( 2 '-naphthyloxy or $4^{\prime}$-phenylphenoxy) at C-3 position and hydrophilic substituents, dialkylaminomethyl at $\mathrm{C}-8$ or $\mathrm{O}-\mathrm{acyl}$ at $\mathrm{C}-7$ positions. The presence of phenyl residue at $\mathrm{C}-3$ position either substituted or non-substituted, resulted in the loss of antiproliferative activity. The presence of chlorine atom at the ortho-position in structure 1 is critical for the biological activity, as meta- or para-substitution 
activities of chromenone derivatives aGainst Cancer CELl

Table I

Antiproliferative activities (GI50 and TGI) of 7-hydroxy-3-aryloxy-2-trifluoromethyl-4H-4-chromenone derivatives I-8 against selected human cancer cell lines

\begin{tabular}{|c|c|c|c|c|c|c|c|c|c|}
\hline \multirow{2}{*}{ Human caocer cell liae } & \multirow{2}{*}{ Values } & \multicolumn{8}{|c|}{ Concentrations $(\mu \mathrm{M})$ for compounds $1-8$} \\
\hline & & 1 & 2 & 3 & 4 & 5 & 6 & 7 & 8 \\
\hline \multirow[t]{2}{*}{ K-562 (Leukemia) } & $\mathrm{GI}_{50}$ & 41.1 & 27.5 & 28.4 & 23.8 & 13.6 & ND* & 28.1 & 9.57 \\
\hline & TGI & $>100$ & 87.1 & 77.8 & 68.1 & 41.8 & ND & $>100$ & 31.2 \\
\hline \multirow[t]{2}{*}{ RPMI-8226 (Leukemia) } & GI50 & 13.5 & 31.5 & 23.4 & 13.6 & 17.0 & ND & 13.9 & 4.60 \\
\hline & TGI & 50.6 & $>100$ & 53.4 & 56.9 & 51.9 & ND & 46.4 & 29.4 \\
\hline \multirow{2}{*}{$\begin{array}{l}\text { NCI-H226 (Non-small cell } \\
\text { lung cancer) }\end{array}$} & GI50 & 19.9 & 21.3 & 15.0 & 12.8 & 16.2 & 14.4 & 30.9 & 33.4 \\
\hline & TGI & 44.6 & 49.3 & 39.4 & 30.8 & 38.4 & 28.0 & 71.5 & 69.3 \\
\hline \multirow{2}{*}{$\begin{array}{l}\text { NCI-H522 (Non-small cell } \\
\text { lung cancer) }\end{array}$} & GI50 & 13.4 & 13.6 & 17.6 & ND & 14.5 & 16.1 & 14.5 & 8.77 \\
\hline & TGI & 30.8 & 33.7 & 36.7 & ND & 35.7 & 34.0 & 42.6 & 34.9 \\
\hline \multirow[t]{2}{*}{ COLO 205 (Colon cancer) } & GI50 & 21.6 & 15.9 & 16.3 & 17.6 & 14.7 & 21.1 & 20.7 & 17.7 \\
\hline & TGI & 46.4 & 30.0 & 30.5 & 36.9 & 28.0 & 48.3 & 48.5 & 41.7 \\
\hline \multirow[t]{2}{*}{ HCI-116 (Colon cancer) } & GI50 & 16.7 & 17.1 & 13.2 & 12.0 & 12.2 & 25.1 & 17.5 & 13.3 \\
\hline & TGI & 30.3 & 59.4 & 26.0 & 30.9 & 24.6 & 39.8 & 32.6 & 26.2 \\
\hline \multirow[t]{2}{*}{ SK-MEL-5 (Melanoma) } & GI50 & 18.1 & 17.7 & 20.1 & 11.5 & 10.5 & 11.4 & 16.0 & 6.07 \\
\hline & TGI & 35.4 & 37.6 & 39.3 & 27.4 & 25.7 & 24.1 & 33.9 & 20.0 \\
\hline \multirow[t]{2}{*}{ UACC-62 (Melanoma) } & $\mathrm{GI}_{50}$ & 14.1 & 14.8 & 13.7 & 14.8 & 16.6 & 18.3 & 20.6 & 17.4 \\
\hline & TGI & 28.3 & 31.2 & 27.0 & $>100$ & 81.1 & 32.2 & 37.7 & 34.2 \\
\hline \multirow[t]{2}{*}{ OVCAR-3 (Ovarian cancer) } & $\mathrm{GI}_{50}$ & 17.0 & 13.7 & 39.3 & 19.7 & 12.8 & 16.7 & 16.0 & 11.9 \\
\hline & TGI & 38.7 & 34.2 & $>100$ & 43.7 & 30.0 & 30.3 & 30.1 & 27.2 \\
\hline \multirow[t]{2}{*}{ SK-OV-3 (Ovarian cancer) } & Giso & 26.7 & 16.8 & 17.8 & 17.2 & 33.4 & 17.7 & 17.5 & 22.8 \\
\hline & TGI & 61.4 & 32.8 & 37.5 & 35.2 & $>100$ & 32.5 & 46.7 & 46.5 \\
\hline \multirow[t]{2}{*}{ CAKI-1 (Renal cancer) } & GI50 & 14.6 & 10.2 & 27.8 & 21.9 & 10.7 & 16.4 & 19.9 & 17.6 \\
\hline & TGI & 31.2 & 31.6 & 52.4 & 47.3 & 29.1 & 30,0 & 39.9 & 35.3 \\
\hline \multirow[t]{2}{*}{ RXF 393 (Renal cancer) } & $\mathrm{Gl}_{50}$ & 16.6 & 17.8 & 19.7 & 14.9 & 15.1 & 10.8 & 44.9 & 23.5 \\
\hline & TGI & 36.5 & 58.5 & 46.9 & 31.8 & 33.2 & 24.8 & $>100$ & 83.2 \\
\hline \multirow[t]{2}{*}{ DU-145 (Prostate cancer) } & $\mathbf{G I} 50$ & 32.3 & 15.2 & 50.0 & 23.1 & 19.0 & 14.9 & 34.2 & 18.9 \\
\hline & TGI & $>100$ & 30.9 & $>100$ & 46.9 & 61.1 & 37.0 & $>100$ & 45.9 \\
\hline \multirow{2}{*}{$\begin{array}{l}\text { MDA-MB-435 (Breas1 } \\
\text { cancer) }\end{array}$} & GI50 & 17.4 & 15.9 & 17.9 & 14.4 & 16.6 & 17.2 & 17.8 & 13.8 \\
\hline & TGl & 35.6 & 32.1 & 33.9 & 29.5 & 37.2 & 30.9 & 37.4 & 28.3 \\
\hline \multirow[t]{2}{*}{ T-47D (Breasi cancer) } & $\mathrm{GI}_{50}$ & 30.9 & ND & 34.7 & 29.1 & 3.44 & 18.9 & 14.2 & 18.5 \\
\hline & TGI & 87.7 & 22.6 & 92.8 & 63.8 & 37.7 & 41.5 & 67.9 & 46.7 \\
\hline
\end{tabular}

*ND - not determined.

leads to significant decrease of antiproliferative activity. At the same time, the activity of $2^{\prime}, 4^{\prime}$-dichloro derivative was only slightly lower. Introduction of some alkylaminomethyl groups into $\mathrm{C}-8$ position of structure 1 also resulted in the activity decrease. Activities of compounds 2, containing trifluoromethyl group, and 3 were close. Analogs of structure 1, compounds $4-8$ without $\mathrm{CF}_{3}$-group at $\mathrm{C}-2$ position showed decreased activities. Substitution of 2 -naphthyloxy moiety at C-3 position of compounds $4-8$ for 4 '-phenylphenoxy group decreased the activity. Compound 8 demonstrated significant inhibition of many 
Table 2

Cytotoxic activity of 7-hydroxy-3-phenoxy-2-trifluoromethyl-4H-4-chromenone derivatives 1-8 toward selected human cancer cell lines

\begin{tabular}{|c|c|c|c|c|c|c|c|c|c|}
\hline \multirow{2}{*}{ Human cancer celt line } & \multirow{2}{*}{ Values } & \multicolumn{8}{|c|}{ Concentrations $(\mu \mathrm{M})$ for compounds $1-8$} \\
\hline & & 1 & 2 & 3 & 4 & 5 & 6 & 7 & 8 \\
\hline (Non-small cell lung cancer) & $\mathbf{L} \mathbf{C}_{50}$ & $>100$ & $>100$ & 61.9 & 50.0 & $>100$ & 81.6 & 82.3 & 54.2 \\
\hline (Non-small cell lung cancer) & LC 50 & 70.8 & 83.4 & 76.2 & ND & 88.1 & 71.6 & $>100$ & $>100$ \\
\hline COLO 205 (Colon cancer) & LC 50 & 99.3 & 56.7 & 57.0 & 77.3 & 53.4 & $>100$ & $>100$ & 98.0 \\
\hline HCT-116 (Colon cancer) & LCso & 55.0 & $>100$ & 51.0 & 79.4 & 49.6 & 63.1 & 60.7 & 51.7 \\
\hline SF-539 (CNS cancer) & $\mathrm{LC}_{50}$ & 66.1 & 55.4 & 57.0 & 59.5 & 74.3 & 58.7 & 81.8 & 76.9 \\
\hline U251 (CNS cancer) & $\mathrm{LC} 50$ & 75.4 & $>100$ & 70.7 & 51.0 & 81.6 & 53.0 & 68.3 & 68.7 \\
\hline M1 4 (Melanoma) & $\mathrm{LC}_{50}$ & 88.6 & 96.6 & 50.9 & 96.4 & 70.8 & 62.2 & 66.8 & 58.5 \\
\hline SK-MEL-5 (Melanoma) & $\mathrm{LC}_{50}$ & 69.2 & 79.8 & 76.8 & 65.1 & 62.6 & 50.8 & 71.7 & 54.2 \\
\hline UACC-62 (Melanoma) & LCso & 57.0 & 65.8 & 52.9 & $>100$ & $>100$ & 56.7 & 68.9 & 67.2 \\
\hline OVCAR-3 (Ovarian cancer) & $\mathrm{LC}_{50}$ & 88.3 & 84.8 & $>100$ & 97.3 & 70.4 & 55.0 & 56.7 & 62.4 \\
\hline
\end{tabular}

*ND - not determined.

cell lines. Further studies will be performed on the optimization of the active structures $1-8$ found in the present data set.

Supporting Information Available: Pre-screening data for 234 tested compounds and ${ }^{1} \mathrm{H}$ NMR spectra for compounds $1-8$. This material is available free of charge via the Internet at www.yarmoluk.org.ua.

Acknowledgement. We are grateful to the $\mathrm{Na}$ tional Cancer Institute of the USA for generous support of our programs.

\section{А. О. Приходько, Г. Г. Дубініна, В. П. Хиля, С. М. Ярмолюк}

Антипроліферативна активність деяких похідних 7-гідрокси-3арилоксм-2-трифторметил-4Н-4-хроменону проти 60 ліній клітин раку людини

Резюме

Синтезовино 234 похідних 7-гідрокси-3-арилокси-2-трифторметил-4Н-4-хроменону та досліджено їхню протиракову активність на лініях ракових клітин люоини у Національному Інституті Раку (СIIA). Сполуки $1-8$ виявили високу цитостатичну та читотоксичну активність $\left(G I_{50} 3,44-41,1\right.$ мкM ma $L C_{50}$ віd 49,6 мкM). Обговорюеться взасмозв'язок між структурою тестованих сполук та їхнью проліферативною активністю.

\section{А. А. Приходько, Г. Г. дубинина, В. П. Хиля, С. Н. Ярмолюк}

Антипролиферативная активность некоторых производных 7 гидрокси-3-арилокси-2-трифторметил-4Н-4-хроменона против 60 линий раковых клеток человека

\section{Резюме}

Синтезированы 234 производных 7-гидрокси-3-арилокси-2-трифторметил-4Н-4-хроменона и исследована их противораковая активность на линиях раковых клеток иеловека в Национальном Институте Рака (СШНА). Соединения $1-8$ проявити высокую читостатическую и цитотоксическую активность $\left(G_{50} 3,44-41,1\right.$ мкM и LC 50 om 49,6 мкM). Обсуждается взаимосвязь мехду структурой тестированных соединений и их пролиферативной активностью.

\section{REFERENCES}

1. Cotelle N. Role of flavonoids in oxidative stress // Curr. Top. Med. Chem.-2001.-1.-P. 569-590.

2. Van Acker F. A. A., Hageman J. A., Haenen G. R. M. M. Van der Vijgh W. J. F., Bast A., Menge W. M. P. B. Synthesis of novel 3,7-substituted-2-(3',4'-dihydroxyphenyl) flavones with improved antioxidant activity // J. Med. Chem.-2000.43. -P. 3752-3760.

3. Owen R. W., Giacosa A., Hull W. E., Haubner R., Spiegelhalder B., Bartsch $H$. The antioxidant/anticancer potential of phenolic compounds isolated from olive oil // Eur. J. Cancer.-2000.-36.-P. 1235-1 247.

4. Jing Y., Waxman S. Structural requirements for differentiation-induction and growth-inhibition of mouse erythroleukemia cells by isoflavones // Anticancer Res. - 1995.-15.P. 1147-1152.

5. Ren W., Qiao Z., Wang H., Zhu L., Zhang L., Cui Y. Lu. Y., Zhang $Z$., Wang $Z$. Tartary buckwheat flavonoid activities caspase 3 and induces HL-60 cell apoptosis // Meth. Find Exp. Clin. Pharmacol.-2001.-23._P. 427-432.

6. Wenzel $U$., Kuntz S., Brendel $M$. D., Daniel $H$. Dietary flavone is a potent apoptosis inducer in human colon carcinoma cells // Cancer Res.-2000.-60D.-P. 3823-3831.

7. Wang I. K, Lin-Shiau S. Y., Lin J. K. Induction of apoptosis by apigenin and related flavonoids through cytochrome $c$ release and activation of caspase- 9 and caspase- 3 in leukemia HL-60 cells // Eur. J. Cancer.-2000.-35.-P. 1517--1525.

8. Ko W. G., Kang T. H., Lee S. J., Kim N. Y., Kim Y. C., Sohn D. H., Lee B. H. Polymethoxyflavonoids from Vitex rotundifolia inhibit proliferation by inducing apoptosis in human myeloid leukemia cells // Food Chem. Toxicol,-2000.-38.P. $861-865$. 
9. Traxler P., Green J., Mett H., Sequin U., Furet P. Use of a pharmacophore model for the design of EGFR tyrosine kinase inhibitors: isoflavones and 3-phenyl-4(1H)-quinolones $/ / \mathrm{J}$. Med. Chem.-1999.-42.-P. 1018-1026.

10. Geahlen R. L., Koonchanok N. M., McLaughlin J. L., Pratt D. E. Inhibition of protein-tyrosine kinase activity by flavonoids and related compounds // J. Nat. Products.-1989.52.-P. 982-986.

11. Cushman M., Nagarathnam D., Burg D. L. Geahlen R. L Synthesis and protein-tyrosine kinase inhibitory activities of flavonoid analogues // J. Med. Chem.-1991.-34.-P. 798806.

12. Srivastava $A . K$. Inhibition of phosphorylase kinase, and tyrosine protein kinase activities by quercetin // Biochem. and Biophys. Res. Communs. $-1985 .-131 .-$. P. $1-5$.

13. Lopez-Lazaro M., Martin-Cordero C., Toro M. V., Ayuso M. J. Flavonoids as DNA topoisomerase I poisons // J. Enzyme Inhib. Med. Chem.-2002.-17.-P. 25-29.

14. Lopez-Lazaro M., Martin-Cordero C., Ayuso M. J. Two new flavonol glycosides as DNA topoisomerase 1 poisons $/ / \mathrm{Z}$ Naturforsch. -2000 - -55.-P. 898-902.

15. Gamet-Payrastre L., Manenti S., Gratacap M. P., Tulliez J., Chap H., Payrastre B. Flavonoids and the inhibition of PKC and PI 3-kinase // Gen. Pharmacol.-1999.-32.-P. 279286.

16. Agullo G., Gamet-Payrastre L., Manenti S., Viala C., Remesy C., Chap H., Payrastre $B$. Relationship between flavonoid structure and inhibition of phosphatidylinositol 3-kinase: a comparison with tyrosine kinase and protein kinase $\mathrm{C}$ inhibition // Biochem. Pharmacol.-1997.-53.-P. 1649-1657.

17. Ferriola $P$. C., Cody V., Middleton E., Jr. Protein kinase C inhibition by plant flavonoids. Kinetic mechanism and structure-activity relationships // Biochem. Pharmacol.-1989.38.-P. $1617-1624$.

18. Sarno S., Moro S., Meggio F., Zagotto G., Dal Ben D.
Ghisellini P., Battistutta R., Zanotti G., Pinna L. A. Toward the rational design of protein kinase casein kinase- 2 inhibitors // Pharmacology and Therapeutics. - 2002.-93.-P. 159168.

19. Casagrande $F$, Darbon $J$. $M$. Effects of structurally related Favonoids on cell cycle progression of human melanoma cells: regulation of cyclin-dependent kinases CDK2 and CDK1 // Biochem. Pharmacol.-2001.-61.-P. 1205-1215

20. Losiewicz M. D., Carlson B. A., Kaur G., Sausville E. A. Worland $P . J$. Potent inhibition of $\mathrm{CDC} 2$ kinase activity by the flavonoid L86-8275 // Biochem, and Biophys. Res. Communs. - 1994.-201.-P. 589-595.

21. Thomas J. P., Tutsch $K$. D., Cleary J. F, Bailey $H$. $H$., Arzoomanian R., Alberti D., Simon K., Feierabend C., Binger $K$, Marnocha $R$., Dresen $A$., Wilding $G$. Phase I clinical and pharmacokinetic trial of the cyclin-dependent kinase inhibitor flayopiridol // Cancer Chemother. Pharmacol.-2002. - 50.P. $465-472$.

22. Vasilev S. A., Luk'yanchykov M. S., Molchanov G. I., Turubarov $V$. D., Khilya V. P. Synthesis and biological properties of 3-phenoxychromones and 3-phenoxy-4-hydroxy7-methoxycumarine // Khim.-pharm. zhurn.-1991.-7.P. 34-38.

23. Arkhipov V. V., Smirnov M. N., Khilya V. P. Chemistry of modified flavonoids. 19. Synthesis of phenoxyl analogs of isoflavone // Chem. Heterocycl. Comp.-1997.-359.P. $598-603$.

24. Garazd M. M., Arkhipov V. V., Proskurka N. K., Khilya V. P. Chemistry of isoflavonoid hetero analogs. 23. Synthesis of aminoacyl derivatives of 3-phenoxychromone // Chem. Heterocycl. Comp. - 1999.-384.-P. 744-748.

УдК $547.745: 576.385 .5$ Надійшла до редакціі 10.03.04 